\title{
A CASE WITH TUMORS IN THE FOURTH VEN- TRICLE OF THE BRAIN, UNACCOMPANIED BY SPECIAL NERVOUS SYMPTOMS.
}

\author{
BY H. D. SCHMIDT, M.D.,
}

PATHOLOGIST TO THE CHARITY HOSPITAL OF NEW ORLEANS.

$\mathrm{T}$

$\mathrm{HE}$ special interest which the following case presents will be found in the presence of two tumors, very unequal in size, but symmetrically seated in the fourth ventricle of the brain, and unaccompanied by special nervous symptoms.

The patient, a negro, 30 years of age, who had been affected with secondary syphilis, was admitted to the hospital in the latter part of January, 1882 , laboring, at that time, under an attack of croupous pneumonia, dating from the 3 Ist of January. When admitted, the upper and middle lobes of his right lung were found to be hepatized; there was a sound of dulness over the area of the heart, unaccompanied by any murmur. His mind was apparently dull, not being able to answer the questions put to him in a satisfactory manner, a condition which, however, soon disappeared, to be followed by perfect clearness of the intellect, remaining until death. From the time of his admittance the temperature of his body gradually rose to ro $4^{\circ}$ until January 28 th, when it commenced to fall, reaching its normal standard on January 29 th, and leaving the pulse at roo beats per minute. On January 3 Ist, the temperature commenced to rise again, reaching $102^{\circ}$ on February 4 th, and ranging from this time until near death between $101^{\circ}$ and $103^{\circ}$.

As the remaining phenomena observed in this case are such as generally accompany fatal cases of pneumonia, and appear not to 
have stood in any direct relation with the tumors found in the fourth ventricle, I shall pass them over, stating merely that on February I 4 th the temperature commenced again to decline, reaching $97^{\circ}$ on the morning of February 15 th. The body of the patient was then covered with cold perspiration, the respiration hurried, the pulse weak and rapid, but the mind still clear. Death occurred suddenly about noon. There were no special symptoms of nervous origin, such as vertigo, vomiting, headache, paralysis, etc., observed in this case during the course of the disease.

The condition of the organs, as revealed by the autopsy, was that generally met with in fatal cases of croupous pneumonia, accompanied by pericarditis. Thus, the upper and middle lobes of the right lung were found in a state of gray hepatization, the left lung œdematous, and the pericardium filled with nearly a pint of purulent liquid. In the fourth ventricle of the brain the above-mentioned tumors were discovered accidentally; the brain itself presented no other changes indicating disease, but appeared perfectly normal.

The larger one of the tumors, measuring $25 \mathrm{~mm}$. in length, $\mathrm{I}_{3}$ $\mathrm{mm}$. in breadth, and $\mathrm{ro} \mathrm{mm}$. in thickness, was attached to a ribbon-like, lamellar pedicle, $7 \mathrm{~mm}$. broad, which, in a vertical

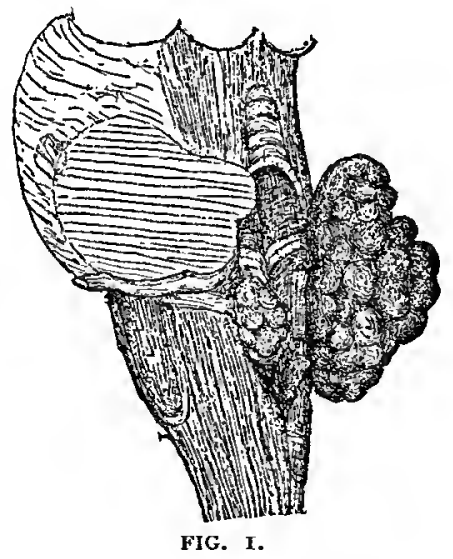

direction, arose from the posterior surface of the right restiform body, forming a part of the sinus rhomboideus, or fourth ventricle (fig. I). The tumor was lobulated, as the drawing shows, and presented the color of the brain. The lamella, or pedicle, was, 
as the subsequent microscopical examination showed, formed by the so-called ponticulus (Henle), and by a portion of the stratum zonale adjoining the latter toward the median line of the ventricle. The smaller tumor, measuring $8 \mathrm{~mm}$. in length, and $3 \mathrm{~mm}$. in thickness, arose, almost opposite, from the same place on the left restiform body; it had no pedicle, but was seated upon the medulla oblongata, being also connected with the ponticulus and stratum zonale. The larger tumor, resting upon the posterior surface of the right restiform body, and thus filling up a considerable portion of the ventricle, was, in its upper surface, pressed upon by the right tonsil of the cerebellum, the pressure being transmitted to the restiform body and adjoining parts. With the smaller tumor this pressure must have been inconsiderable.

The examination of thin sections, both of the tumors and the medulla oblongata, showed that the neoplasm consisted of bundles of very fine fibrillæ, most intricately interwoven in various directions. In the meshes of this fibrillar interlacement a considerable number of small round or oval nuclei of $\frac{5}{1000} \mathrm{~mm}$. in diameter,

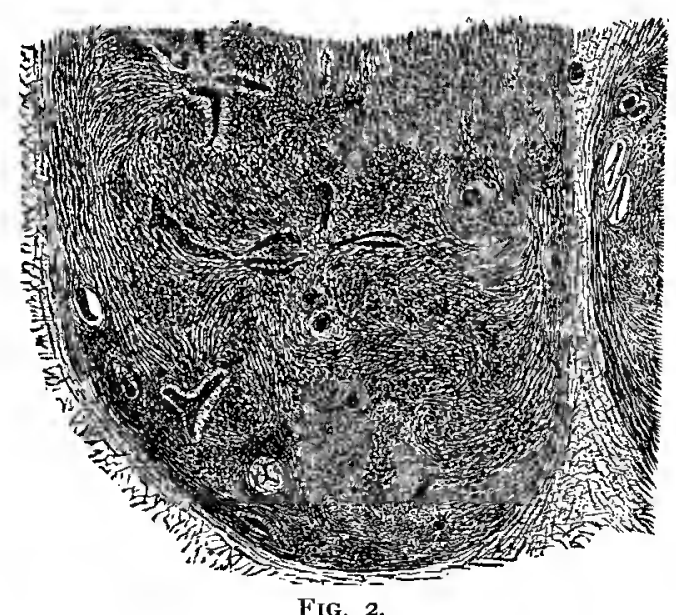

were embedded in the form of numerous groups, representing minor centres, in each lobule of the tumor; around and between these nuclei the fibrillar bundles were arranged in a general manner (fig. 2), while, at the same time, finer subordinate bundles passed, in the form of a plexus, between the nuclei. As the fibrillar element-if stroma it may be called-of this neoplasm 
bears the distinct character of the fibrillæ of the neuroglia, and as no spindle-shaped cells were observed in the tumor, the latter evidently represents a so-called glioma of Virchow. The arrangement of these fine fibrillar bundles is of the most intricate nature ; they are obseryed to pursue almost always a more or less circular course in their interlacement, crossing in every direction. The nuclei contained within their meshes appear to be free-I have, at least, not been able to detect any protoplasm surrounding them; the majority of them are small and round, of the diameter mentioned above, but there are also observed a considerable number slightly larger and of an oval form.

In the sections made through the medulla oblongata and tumors the origin of the latter from the stratum zonale and ponticulusthe latter forming a part of the stratum-is distinctly observed. In that portion of the stratum zonale adjoining the larger tumor, namely, the fibrillæ of the neuroglia are seen to pass from the former into the latter, where they collect into fine bundles, from the interlacement of which the lobules are formed, while the same bundles, arranged in the form of a looser tissue, without lodging any nuclei, serve to connect the individual lobules of the tumor. In that portion of stratum zonale from which the larger tumor arises, a considerable number of small bi-polar and some multipolar ganglion cells are met with, which, however, do not pass into the tumor. The microscopical examination also shows how from this part of the medulla the tissue of the latter has been to a certain extent slightly drawn out and deranged by the tumor, as far back as the nucleus of the pneumogastric nerve, the ganglion cells and nerve fibres of which are observed separated in the form of columns, parallel to the surface, and in a direction toward the origin of the tumor.

The tumors, particularly the larger, are abundantly supplied with blood-vessels filled with blood corpuscles, indicating that they were in a state of congestion during life; the same phenomenon is observed in the vessels of the medulla oblongata. The vessels entering the tumor appear to have been directly derived from the choroid plexus of the fourth ventricle. They are seen to enter the loose interlobular tissue, whence they send their ramifications into the interior of the individual lobules.

As regards the condition of the medulla oblongata itself, I may state that its consistence appeared to be greater than normal. That this was the case became evident during the cutting of the sections by a certain indian-rubber-like toughness, which rendered 
the tissue more difficult to cut than that of a normal medulla, though the microscopical examination revealed no increase in the number of the small nuclei of the neuroglia.

There are two interesting features presented by the above-described neoplasm, viz.: the development and growth of a tumor as large as the one here concerned, through a delicate ribbon-like pedicle ; and, furthermore, the peculiar symmetry observed in the seat of these tumors. It may be presumed that if the patient had lived long enough they would, by a continued growth of the smaller one, have eventually completely filled up and distended the whole ventricle. While in other pediculated tumors the pedicle is usually formed by traction owing to the weight of the tumor, we observe in this case the tumor growing out, and, mor,eover, in an upward direction, from the pedicle, adapting its form to that of the cavity into which it grew. As concerns the symmetry of location of pathological formations and lesions, it has often been observed. I know not whether a satisfactory explanation has ever been rendered of this interesting phenomenon, and regret, for the want of time, not to be able at present to enlarge upon it.

Another interesting feature presented by this case consists in the absence of any special nervous symptoms, though the pressure exerted by the larger tumor upon the adjoining parts must have been considerable. Whether this pressure upon the nucleus of the pneumogastric nerve and the lesion observed in this centre stood in any relationship with the pneumonia, from which the patient died, remains an open question.

\section{Explanation of Illustrations.}

FIG. I.--Representation of the medulla oblongata, with pons and the tumors attached.

Fig. 2.- Representation of a transverse section of the tumor, showing its structure. The fibrillar bundles, highly colored with picro-carmine, are seen crossing another in every direction, while they pursue a circular course around the groups of nuclei; the latter are colored dark-blue with hematoxylin. In the plane of the section the fibrillar bundles, of course, appear cut at various angles, from the transverse, through the oblique, to the almost longitudinal. The vessels in the section are colored highly carmine. Magnified about $5^{\circ}$ diameters. 